\title{
Primary mirror dynamic disturbance models for TMT: Vibration and wind
}

\author{
Douglas G. MacMynowski ${ }^{a}$, M. Mark Colavita ${ }^{b}$, \\ Warren Skidmore ${ }^{c}$ and Konstantinos Vogiatzis ${ }^{c}$ \\ ${ }^{a}$ California Institute of Technology \\ Department of Control and Dynamical Systems, Pasadena CA 91125 \\ ${ }^{b}$ Jet Propulsion Laboratory, California Institute of Technology, Pasadena, CA 91109 \\ ${ }^{c}$ Thirty Meter Telescope Observatory, Pasadena, CA 91125
}

\begin{abstract}
The principal dynamic disturbances acting on a telescope segmented primary mirror are unsteady wind pressure (turbulence) and narrowband vibration from rotating equipment. Understanding these disturbances is essential for the design of the segment support assembly (SSA), segment actuators, and primary mirror control system (M1CS). The wind disturbance is relatively low frequency, and is partially compensated by M1CS; the response depends on the control bandwidth and the quasi-static stiffness of the actuator and SSA. Equipment vibration is at frequencies higher than the M1CS bandwidth; the response depends on segment damping, and the proximity of segment support resonances to dominant vibration tones. We present here both disturbance models and parametric response. Wind modeling is informed by CFD and based on propagation of a von Karman pressure screen. The vibration model is informed by analysis of accelerometer and adaptive optics data from Keck. This information is extrapolated to TMT and applied to the telescope structural model to understand the response dependence on actuator design parameters in particular. Whether the vibration response or the wind response is larger depends on these design choices; "soft" (e.g. voice-coil) actuators provide better vibration reduction but require high servo bandwidth for wind rejection, while "hard" (e.g. piezo-electric) actuators provide good wind rejection but require damping to avoid excessive vibration transmission to the primary mirror segments. The results for both nominal and worst-case disturbances and design parameters are incorporated into the TMT actuator performance assessment.
\end{abstract}

\section{Keywords: Extremely Large Telescopes, Wind, Vibration, Control Systems}

\section{INTRODUCTION}

The primary mirror of the Thirty Meter Telescope (TMT) is composed of 492 segments, with stringent performance requirements on the allowable motion of the out-of-plane degrees of freedom for both seeing-limited and adaptive-optics observations. The segment motion is influenced by both quasi-static (gravity and thermal) and dynamic disturbances, including unsteady wind forces and equipment vibration. Low frequency wind disturbances can be partly attenuated by the primary mirror control system (M1CS). Disturbances due to equipment vibration will be well above the control bandwidth, but may be close to segment support resonant frequencies, with the response influenced by the segment support stiffness and damping. Thus the response to both wind forces and vibration disturbances are influenced by the choice of primary mirror actuators.

With a "soft" voice-coil actuator, stiffness is provided at low frequencies using encoder-feedback to a local servo loop. Because this type of actuator is soft at frequencies above the servo bandwidth, it naturally attenuates the response to disturbances from equipment vibration. However, depending on the gain of the servo loop, it will be less stiff at low frequencies than a "hard" actuator such as a piezo-electric, and therefore the response to low-frequency wind forces may be larger. Thus the choice between actuator technologies is strongly influenced by an understanding of these two dynamic disturbances; this is illustrated in Fig. 1 (using parameters from Sec. 4). The wind disturbance also determines the M1CS global control bandwidth requirement, while the need to avoid resonant excitation near expected vibration frequencies determines the requirement on the resonant frequencies of the segment support assembly (SSA), and therefore on the SSA and actuator stiffness.

This paper presents models for both of these disturbance sources, and explores the response as a function of disturbance and actuator parameters. The wind disturbance assumptions are informed by computational fluid dynamics (CFD) and Gemini data, ${ }^{1-3}$ and the response model applies a von Karman pressure-screen propagating 

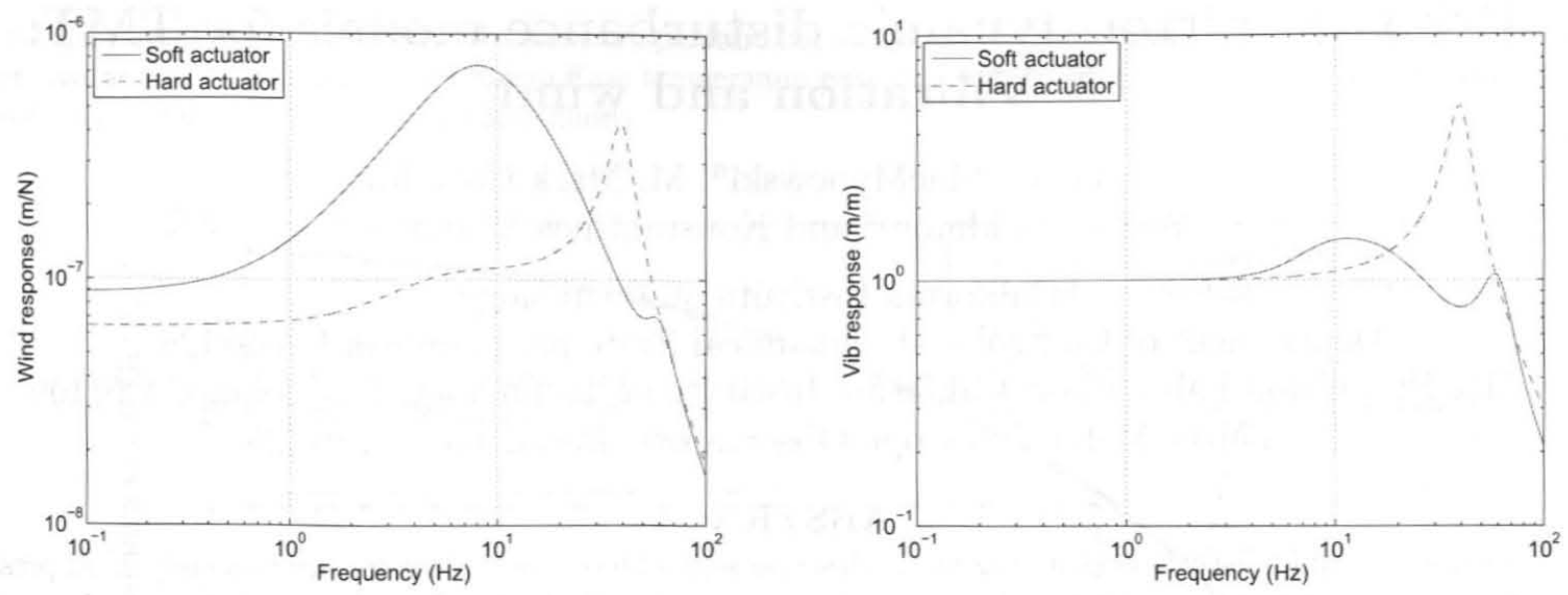

Figure 1. Characteristic response comparison for soft and hard actuators to forces on mirror surface (left), relevant to the wind response, and displacement at segment base (right), relevant to vibration response. In each case the mirror surface piston response is plotted for a segment mounted on a rigid base, using parameters from Sec. 4 . A soft actuator has lower vibration response near $30 \mathrm{~Hz}$, but larger wind response (wind energy is mostly below $1 \mathrm{~Hz}$ ). The details of the soft-actuator response depends on the servo design, while the resonant amplification for the hard actuator depends on the damping (here 10\%). Both actuators have servo loops with integral gain, the small difference in the static stiffness is due to increased compliance not sensed by the collocated encoder used by the servo loop of the soft actuator.

across a structural model of M1 as in [4]. The vibration disturbance assumptions are informed by data taken at the Keck Observatory, and the response computed by applying pier vibrations to the TMT finite element model. One of the dominant tones in the response spectrum at Keck is close to $30 \mathrm{~Hz}$ (due to large induction motors), and since this is close to the segment support resonant frequency, the modeling focuses on this tone in particular.

The modeling information is used to inform the actuator downselect decision by estimating the performance of alternate actuator technologies based on both nominal estimates of all parameters, and worst-case estimates for disturbances, actuator performance, and structural design performance.

Performance estimates for seeing-limited observations are quoted in normalized point-source sensitivity (PSSN). ${ }^{5}$ These are typically slightly more stringent requirements for the M1 dynamic errors than the uncorrectable wavefront error relevant for adaptive optics (AO). The PSSN allocation for segment out-of-plane dynamic displacement due to wind and vibration is 0.9935 .

\section{M1 WIND LOADS AND RESPONSE}

\subsection{Wind disturbance characteristics}

Some wind over M1 is necessary to flush the dome and manage dome and mirror seeing. The target wind speed results from a trade-off between the thermal effects and M1 wind-buffeting; the optimum speed is roughly $1 \mathrm{~m} / \mathrm{s}$. This target is maintained by controlling the vent opening, as a function of both the external wind speed and orientation. CFD analysis has been performed at different telescope orientations relative to the wind and different vent openings, in order to determine the ratio of internal and external wind speeds at each condition. This is combined with statistical estimates of external wind speed, wind orientation, and observing orientation. ${ }^{6}$ The resulting probability distribution for the mean M1 wind speed $\bar{u}$ is shown in Fig. 2 for a $1 \mathrm{~m} / \mathrm{s}$ target velocity. The result of this strategy is that the mean wind velocity is near $1 \mathrm{~m} / \mathrm{s}$ roughly half the time. The performance analysis below thus focuses on this representative case.

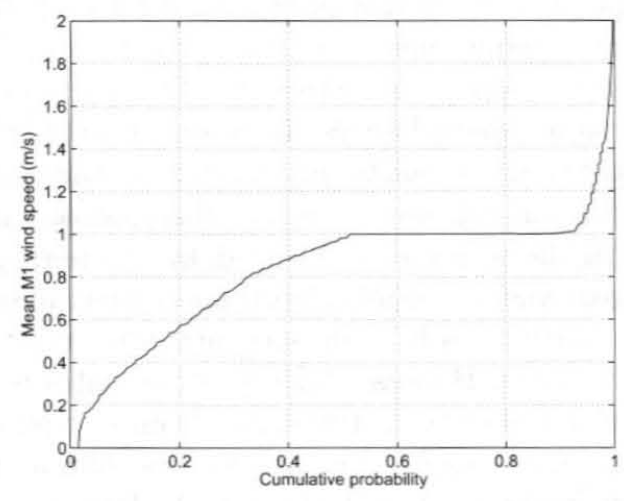

Figure 2. Estimated probability distribution of wind speed over M1, assuming a target speed of $1 \mathrm{~m} / \mathrm{s}$ controlled by the vent opening. At low external wind speeds, the M1 wind speed is less than $1 \mathrm{~m} / \mathrm{s}$ with fully-open vents; at high external wind speeds the M1 wind speed exceeds $1 \mathrm{~m} / \mathrm{s}$ with closed vents. 
Static forces are completely corrected by the integral control of M1CS; it is only the unsteady (turbulent) forces that lead to residual segment dynamic response. The turbulence is characterized by the rms pressure and spatial and temporal spectra (or equivalently correlation information).

The rms pressure is obtained from unsteady CFD calculations. At a $1 \mathrm{~m} / \mathrm{s}$ mean wind speed, the rms unsteady differential pressure across the mirror is roughly $0.21 \mathrm{~Pa}$ (at Mauna Kea air density of $\rho=0.819 \mathrm{~kg} \mathrm{~m}^{-3}$ ). If this is expressed through Bernoulli in terms of an effective unsteady wind speed, ${ }^{1,7}$ this corresponds to $u_{\text {eff }}=0.72 \mathrm{~m} / \mathrm{s}$ or turbulent intensity of $25 \%$. (Note that $p=\frac{1}{2} \rho u^{2}$ assumes viscous losses are negligible, and so does not hold in the boundary layer or in regions of separated flow, hence estimates of unsteady pressure from unsteady wind speeds can be unreliable near M1.)

With a frozen turbulence assumption then the spatial and temporal spectra are related. Measurements at Gemini ${ }^{1-3}$ are consistent with a von Karman spectrum in both temporal spectra and (spatial) structure functions. The outer scale ${ }^{*}$ at Gemini is of order $8-10 \mathrm{~m}$. This is consistent with the turbulence being caused by flow entering through the aperture, or through the vents, or through interaction with M1. Thus it is not obvious how to scale the outer scale from Gemini measurements to TMT. At TMT, the aperture and M1 are both of order $30 \mathrm{~m}$, while the vent height is of order $5 \mathrm{~m}$. Recent unsteady CFD for TMT suggests that it is the interaction of the flow with M1 that is the dominant source of turbulent forces on M1, rather than the primary turbulence being created by flow passing through the vents and then being advected across M1; this is consistent with hypotheses from Gemini. ${ }^{2}$ This would suggest an outer scale of $30 \mathrm{~m}$ for turbulence at TMT. However, to be conservative, we assume below that $75 \%$ of the turbulent energy has an outer scale of $5 \mathrm{~m}$ and the remaining $25 \%$ has an outer scale of $30 \mathrm{~m}$. This is chosen to be consistent with the average distribution of flow speeds over M1 due to vents versus aperture (that is, with vents closed at a median external wind speed, the flow speed over M1 would be roughly $25 \%$ of the value with vents open).

The assumed temporal power spectrum is thus of the form

$$
\Phi_{p}(f)=\left\langle p^{2}\right\rangle\left\{\alpha \Phi\left(f, L_{v}\right)+(1-\alpha) \Phi\left(f, L_{a}\right)+\beta \Phi\left(f, L_{s}\right)\right\}
$$

where the 1-D von Karman pressure spectrum as a function of outer scale, normalized to unit rms amplitude, is $^{7}$

$$
\Phi\left(f, L_{0}\right)=\left(\frac{2 \sqrt{\pi}}{3 \Gamma(2 / 3) \Gamma(5 / 6)}\right) \frac{L_{0} / \bar{u}}{\left[1+\left(L_{0} f / \bar{u}\right)^{2}\right]^{7 / 6}}
$$

$\alpha$ is the fraction of turbulence associated with the vents, $\beta$ is a parameter to allow added conservatism associated with small-scale turbulence introduced by flow passing over structural members not resolved by the CFD, and $L_{v}, L_{a}$ and $L_{s}$ are the length scales associated with vents, aperture, and the additional structural turbulence respectively. There is some evidence from a few cases of Gemini data to support the form of eq. (1). Nominally we assume $\beta=0$, however, to understand the robustness of actuator choices against uncertainty in our understanding of wind, a worst-case model of $\beta=0.2, L_{s}=0.5 \mathrm{~m}$ has been included in analyses. (Note that this parameterization is chosen to increase the rms pressure in addition to adding short-length-scale turbulence.)

\subsection{Wind response modeling}

Given confidence in unsteady CFD modeling, a possible strategy for predicting wind response is simply to apply the differential pressures directly from CFD to the mirror surface in a structural model. To the extent that the CFD is correct, this would give the correct spatial correlation behavior, temporal spectrum, spatial variability of pressure, and spatially non-uniform mean wind speed. However, there are three advantages to developing a parametric model that has roughly the right characteristics. First and most important is that it allows a scaling to predict performance at different conditions from those at which unsteady CFD has been conducted. Second, it allows us to understand how different parameters influence the performance, and potentially to modify our assumptions regarding some parameters if there are some aspects of the CFD solution that we are less confident in than others. And third, it easily allows for simulations of arbitrary time-length, to better understand statistics of closed-loop behavior when the control bandwidth is low and hence convergence time is long.

The basic approach used to predict the segment response given the parameters of the wind model is similar to that used in simulating performance of AO systems. A pressure screen is generated that has the correct 2-D von Karman spatial statistics, and this screen is propagated in the time domain across M1. At each time step,

\footnotetext{
* see [7] for a discussion on the definition of outer scale.
} 

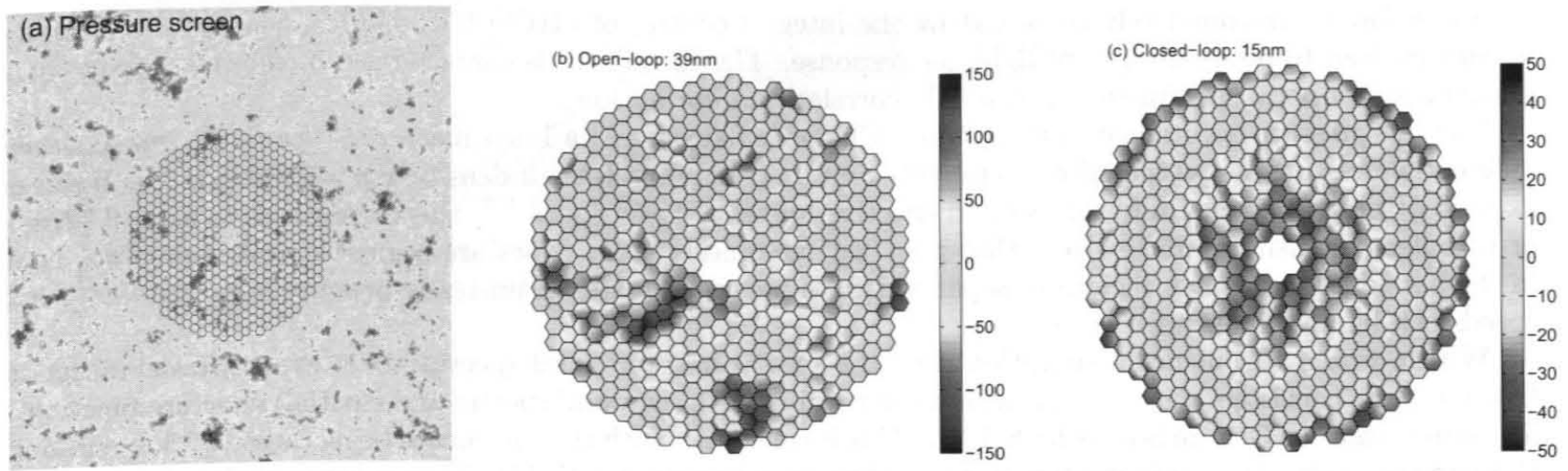

Figure 3. Illustration of modeling process, and typical M1 response to $1 \mathrm{~m} / \mathrm{s}$ wind, in nm. Representative pressure screen (left), mirror response without control (center), and with $1 \mathrm{~Hz} \mathrm{M1CS}$ control bandwidth (right); focus-mode bandwidth here is $0.1 \mathrm{~Hz}$. Global piston, tip and tilt are projected out of the response. The residual rms surface response is 39 and $15 \mathrm{~nm}$ rms respectively; in the controlled case, the focus-mode amplitude is $14 \mathrm{~nm}$ rms and non-focus-mode residual rms surface response is $6 \mathrm{~nm}$.
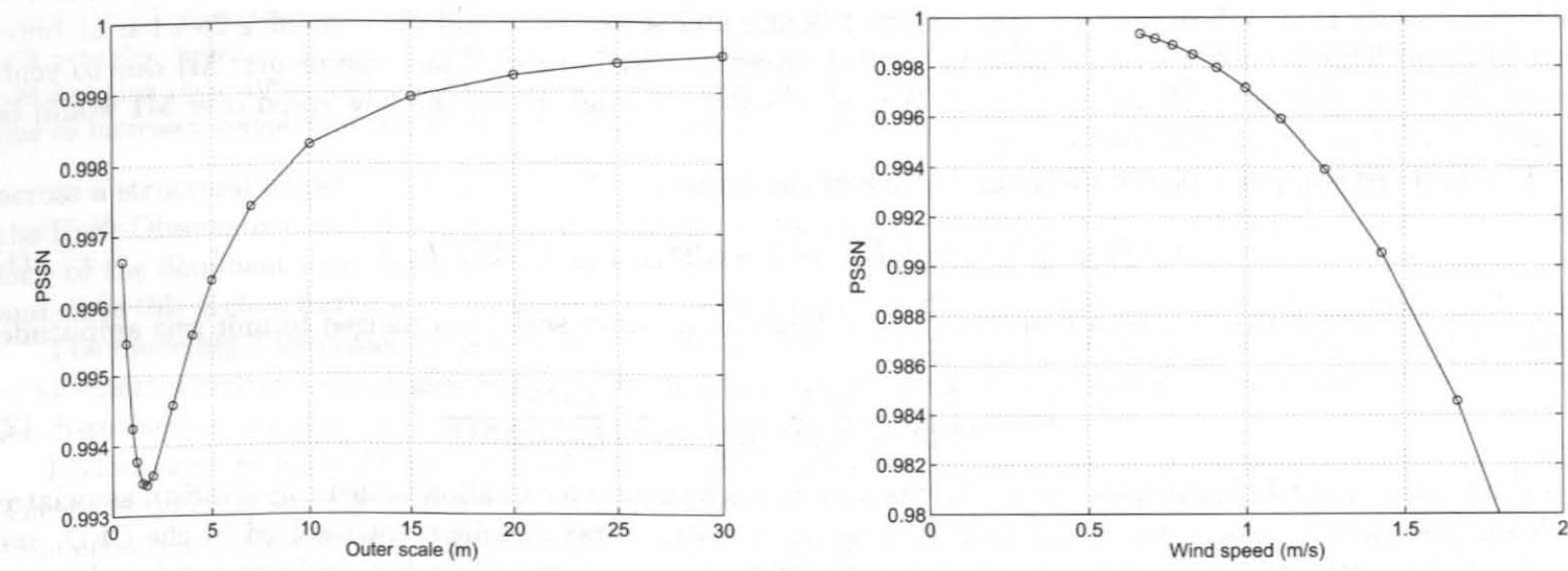

Figure 4. Performance as a function of wind parameters; outer scale (left) and mean wind speed over M1 (right). The outer scale simulation assumes a $1 \mathrm{~m} / \mathrm{s}$ mean wind speed, rms pressure of $0.21 \mathrm{~Pa}$, and $1 \mathrm{~Hz} \mathrm{M} 1 \mathrm{CS}$ control bandwidth. Wind speed simulation assumes that the pressure scales with $u^{2}$, and $75 \%$ of pressure has outer scale $5 \mathrm{~m}, 25 \%$ has outer scale $30 \mathrm{~m}$.

the force and moments on each segment are computed, and the segment responses due to structure and control system dynamics are obtained. In order to obtain a reasonable assessment of performance, several different random seeds for the pressure screen are required. The modeling process is illustrated schematically in Fig. 3.

The wind parameters in the model include the mean wind speed (assumed uniform across the mirror), the rms pressure (also assumed uniform rather than spatially variable), and the outer scale(s) and turbulent energy fraction for the wind turbulence, as in Eq. (1). From Fig. 2, the wind speed is close to the target wind speed much of the time, and thus analysis will concentrate on these conditions.

The response predicted by this approach differs from the response predicted if the unsteady CFD pressures are used directly because (i) CFD predicts that essentially all of the turbulence has an outer scale of $30 \mathrm{~m}$, while we are choosing to be more conservative, and (ii) the unsteady pressure in the CFD solution is primarily due to flow separation around M1, thus the turbulence is not fully-developed, and the spectrum rolls-off faster than a von Karman spectrum; again we are choosing to be more conservative by assuming von Karman spectrum. There is spatial non-uniformity in the CFD in the mean wind speed and the rms pressure, but these do not have a significant performance implication. That is, the differences between the modeling approach taken here and the CFD are not due to limitations of this modeling approach, but due to intentional conservatism in assumptions. 


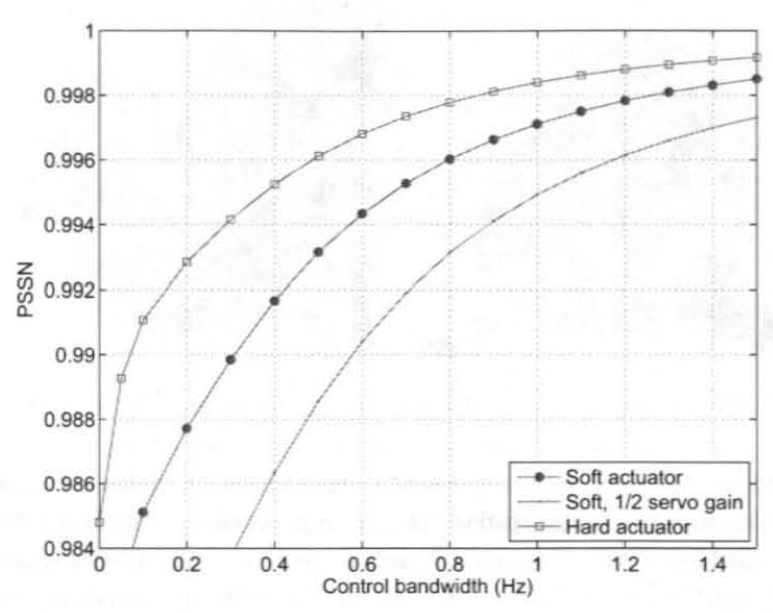

Figure 5. Wind response performance as a function of M1CS bandwidth for nominal assumptions (see Table 1). The TMT segment actuators are "soft" (voice-coil); higher wind rejection is achieved with a hard actuator.

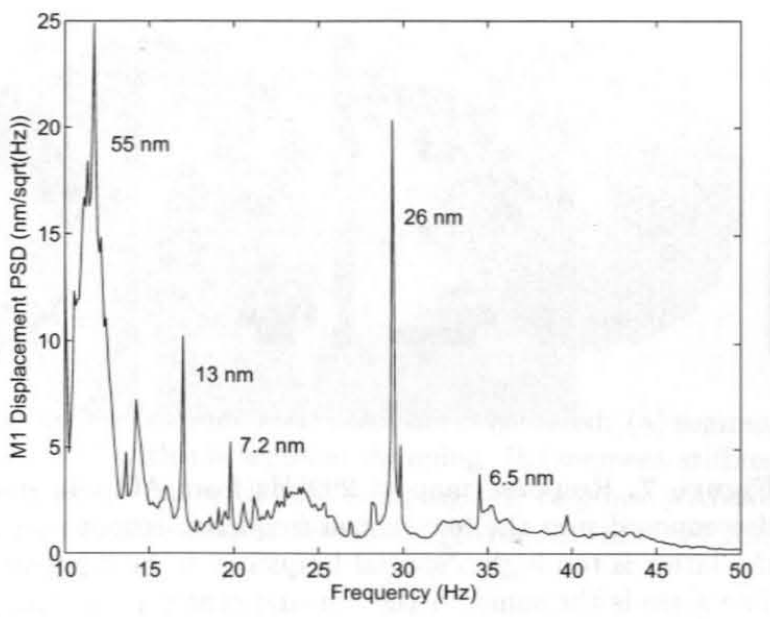

Figure 6. Average vibration spectrum measured on Keck primary mirror, rms over 6 accelerometers during observations. The rms surface displacement of various tones is indicated.

In addition to the wind parameters, the M1 segment dynamic response depends on the structural dynamics and the control bandwidth. Most of the wind energy is at frequencies below $1 \mathrm{~Hz}$. Since this is well below the resonant frequencies of the structure, only the static compliance affects the response. If a voice-coil actuator is used, then the finite bandwidth of the actuator servo loops does affect the response (Fig. 1). Modeling suggests that a $1 \mathrm{~Hz}$ bandwidth is achievable for the global M1CS control loop, ${ }^{8}$ with the exception of focusmode. Because the residual segment tip/tilt (that determines seeing-limited performance) and inter-segment edge discontinuities (that determines $\mathrm{AO}$ performance) are both dominated by the high spatial frequency response, reducing the bandwidth on focus-mode does not have a significant affect on performance and we assume a bandwidth of $0.1 \mathrm{~Hz}$ throughout. (This is why the residual pattern in Fig. 3(c) is predominantly focus-mode.)

Figure 4 shows the dependence of the seeing-limited error as a function of wind model assumptions, while Fig. 5 shows the dependence on actuator and control assumptions.

The dependence on outer-scale is significant, with the worst-case outer-scale being equal to the segment diameter; shorter turbulent length-scales are spatially averaged over a segment and hence lead to smaller forces and moments, while longer length-scales result in a greater fraction of the energy at low spatial frequencies that have less performance impact. The rms wavefront error peaks at $15 \mathrm{~nm}$ when the outer scale is roughly the size of a segment, and drops to $8 \mathrm{~nm}$ at a $30 \mathrm{~m}$ outer scale. While AO might correct only half of the response at the smaller outer-scale, most of the response at a $30 \mathrm{~m}$ outer scale would be at spatial frequencies that the $\mathrm{AO}$ system can correct. If the CFD analysis is correct and the turbulence outer-scale is $\sim 30 \mathrm{~m}$, then it is clear that wind turbulence over M1 is not a significant performance concern at the wind speed and M1CS bandwidth assumed here. With our more conservative assumption that much of the turbulence is due to the interaction of the flow with the $5 \mathrm{~m}$ vents, then it is only this turbulence that dominates the residual segment dynamic displacement, and not whatever fraction is assumed at a $30 \mathrm{~m}$ outer scale.

The dependence on M1 wind speed is also quite significant. A higher wind speed both means that more of the energy is at higher frequencies where M1CS has less reduction, and that the rms pressure is higher.

Fig. 5 illustrates the dependence on M1CS control bandwidth, as well as on actuator assumptions. The hard actuator has better wind rejection. If only half the assumed servo gain can be achieved (see [8]), the performance would still be acceptable at a $1 \mathrm{~Hz}$ M1CS global control bandwidth, however, if both the servo gain and the global control bandwidth are decreased, then the segment dynamic displacement due to wind could become a significant performance driver. (If this were the case in operation, the target M1 wind speed could be reduced, giving a small increase in dome and mirror seeing but reduced segment wind response.) 

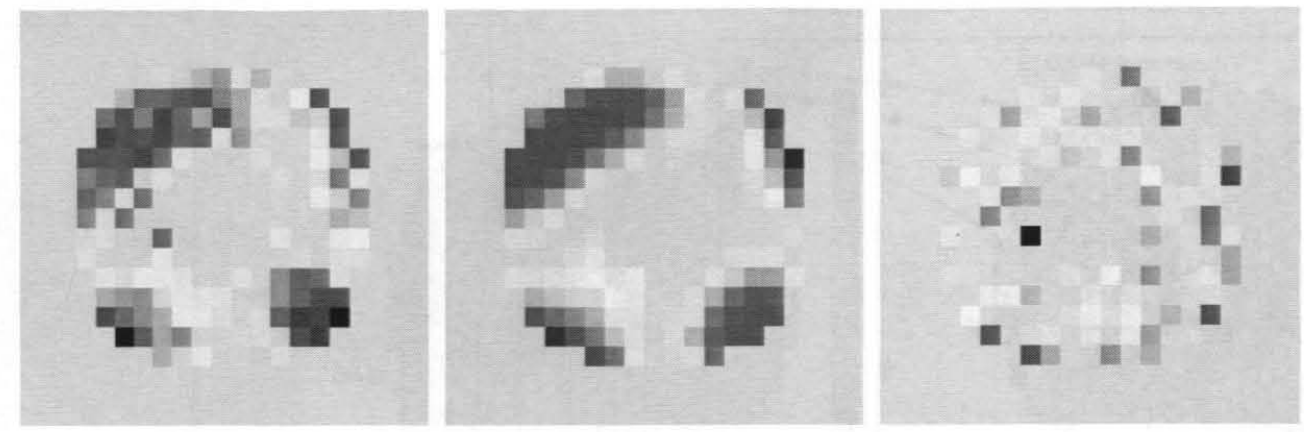

Figure 7. Response map at $29.6 \mathrm{~Hz}$ from AO DM data. Image on the left is a representative snapshot, which can be decomposed into the low-spatial-frequency response in the middle and the high-spatial-frequency response on the right; the latter is too high a spatial frequency to be segment motion, and is of the correct amplitude to be atmospheric noise. The scale is the same on the left and center plots, the scale is magnified by a factor of two on the right to illustrate the spatial pattern.

\subsection{Vibration environment}

\section{VIBRATION}

While equipment vibration is an important source of dynamic response for TMT, the uncertainty in quantifying the amplitude is much higher than it is for wind. Vibrations can be observed in high resolution measurements at Keck Observatory, including narrowband tones near $\sim 29.6 \pm 0.2 \mathrm{~Hz}$ associated with large induction motors; see Fig. 6. Most of the wind response is at frequencies well below the $\mathrm{AO}$ control bandwidth, so that any motion that is spatially correctable by AO can be corrected. However, this is not true for equipment vibration, due to the higher temporal frequencies.

TMT intends to use best engineering practices on source mitigation; choosing low-vibration equipment, locating vibration sources away from the telescope where practical, and isolating equipment from the foundation. Our nominal assumption is that TMT will have half of Keck's vibration amplitude if segment design decisions were comparable (this is an informed guess from Keck experts on how much better could have been done if they could start from scratch). Because this is a rather uncertain assumption, we also analyze performance with a worst-case assumption of TMT having the same vibration amplitude as Keck. Note that the vibration response depends on segment resonant frequencies and actuator design decisions. Thus we choose a vibration source amplitude at the pier that gives half of the Keck vibraiton when we choose segment design parameters for TMT to be consistent with Keck; see the modeling section below for details.

Also note that the amplitude of the $29.6 \mathrm{~Hz}$ tone on the mirror cell at Keck is roughly half the vibration amplitude of the segment surface. This is consistent with the expected resonant amplification from the (measured) $43 \mathrm{~Hz}$ piston resonance of the Keck M1 segments. In addition to the mirror cell and the mirrors themselves, we have also measured vibration amplitudes at Keck on the pier and Nasmyth platforms. However, it is not clear what the dominant transmission path for vibrations is at Keck, or will be at TMT. For modeling here we apply disturbances at the pier and implicitly assume that the characteristic response patterns will not depend significantly on the source or transmission path. Note that this lack of knowledge is why we have chosen to reference our expected vibration specification to the Keck primary mirror, rather than to the Keck pier vibration.

A second piece of useful information regarding M1 vibration at Keck is the spatial pattern of the $29.6 \mathrm{~Hz}$ tone. This can be obtained from AO data. A $100 \mathrm{~Hz}$ bandwidth case was analyzed, so that the AO system is correcting much of the M1 vibration, and the deformable mirror (DM) actuator commands can be used to understand the spatial pattern of vibration. A representative snapshot of the $29.6 \mathrm{~Hz}$ DM response is shown in the left-most panel of Fig. 7. This can be decomposed into the sum of the other two patterns in Fig. 7: a spatially-smooth pattern dominated by astigmatism, and a high-spatial-frequency pattern that is at too high a spatial-frequency to be caused by uncorrelated segment motion, and is presumably the atmospheric response at this frequency. (The amplitude of this pattern is consistent with that expected from the atmosphere based on the signal-to-noise ratio in the temporal spectrum.) 

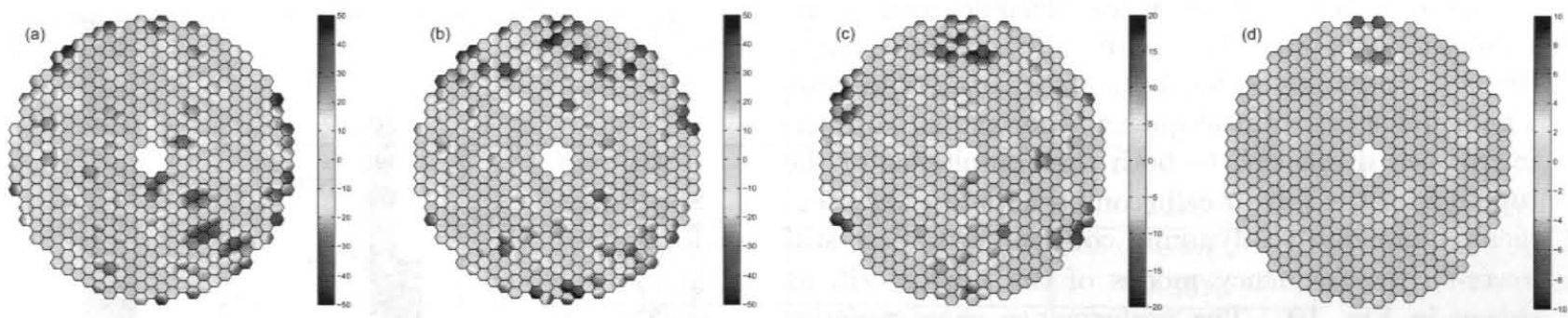

Figure 8. Response map at $29.6 \mathrm{~Hz}$ for TMT model with $10 \mathrm{~nm}$ rms pier vibration. Four cases are considered: (a) segment stiffness set to match segment resonant frequency of Keck ( $43 \mathrm{~Hz}$ piston) with $1 \%$ segment damping, (b) segment stiffness of TMT with a hard actuator with no additional damping, (c) same as (b) but with $10 \%$ damping, (d) response with soft actuator. Note that axes scale is changed on last two plots. rms surface responses are $13 \mathrm{~nm}, 15 \mathrm{~nm}, 5.6 \mathrm{~nm}$ and $2 \mathrm{~nm}$ respectively; the corresponding PSSN is $0.9976,0.9932,0.9994$ and 1.0000 .
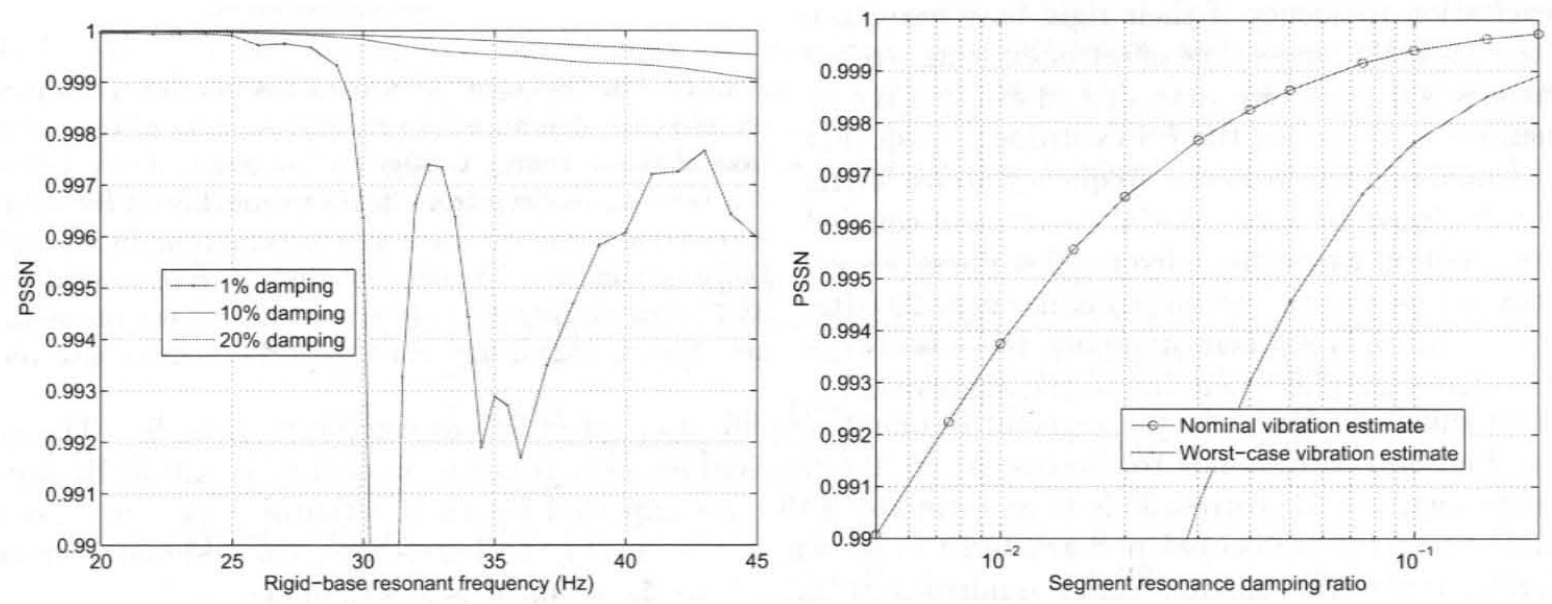

Figure 9. Dependence of vibration response on nominal (rigid-base) segment resonant frequency (left) and segment damping ratio (right). The latter plot also includes the effect of a worst-case assumption of doubling the vibration levels relative to the nominal assumptions.

\subsection{Vibration response}

The Keck M1 response is thus relatively spatially smooth. To predict the response of TMT to this vibration tone, we use the TMT finite element model with 3000 modes extracted (up to $60 \mathrm{~Hz}$, and with the static correction from truncated dynamics included), include the dynamics of the 492 mirror segments, and compute the surface response due to an applied pier vertical displacement. If the segment parameters are chosen comparable to those at Keck, with the segment piston resonance at $43 \mathrm{~Hz}$, then (i) the segment dynamic amplification is comparable to that observed at Keck, with the segment surface motion roughly double the mirror cell motion, and (ii) the spatial correlation length in the TMT response is comparable to that observed in the Keck DM data; see Fig. 8(a). The spatial correlation length of the response is driven by the typical length scales of the structural modes of the mirror cell that are excited at this frequency. This case is used to choose the pier vibration amplitude required to give a vibration amplitude (at the mirror-cell or segment surface) half of that observed at Keck.

Next, consider the effect of changing the segment support resonant frequency and damping. The surface displacement maps for several additional cases are shown in Fig. 8, and the parametric performance dependence shown in Fig. 9. Decreasing the segment resonant frequencies from the Keck value of $43 \mathrm{~Hz}$ to $38 \mathrm{~Hz}$ changes the characteristics of the response. Rather than simply increasing the segment dynamic amplification, the segments now act as tuned-vibration-absorbers on the mirror cell, increasing the impedance seen by the source, and thus reducing the mirror cell vibration amplitude. However, because more of the response is due to segment dynamic amplification rather than mirror cell structural modes, there is larger high-spatial-frequency content to the response, and thus while the overall rms surface response does not increase greatly, the performance (PSSN) is significantly worse. 
Much of this decrease in performance can be attributed to the fact that the nominal $38 \mathrm{~Hz}$ resonant frequency assumes segments mounted on a rigid base. The segment resonant frequency when mounted to the mirror cell drops due to both the compliance of the top layer of the mirror cell (comparable for every segment), and due to dynamic coupling with less stiff lower-spatial-frequency modes of the mirror cell, as shown in Fig. 10. The performance characteristics as a function of segment resonant frequency shown in Fig. 9(a) are due to the segment piston resonances dropping close to the vibration excitation frequency if the rigid-base resonance is in the $34-36 \mathrm{~Hz}$ range, and the segment tip/tilt resonances dropping close to the excitation frequency if their rigid-base resonance is in the $31-32 \mathrm{~Hz}$ range. The effect of exciting tip/tilt resonances is more severe than that of exciting piston resonances; in this case, the PSSN drops to 0.98. For any nominal segment resonant frequency above $30 \mathrm{~Hz}$, there will always be some spatial patterns of coupled segment motion where the mirror-cell stiffness is such that this pattern has a system resonance near $29.6 \mathrm{~Hz}$, and thus with light segment damping, there is always some dynamic amplification due to the segments.

With sufficient damping, the segment resonant amplification effect is less significant (Fig. 9). The spatial map in Fig. 8(c) corresponds to the case where the nominal segment resonant frequency is still $38 \mathrm{~Hz}$ but with $10 \%$ damping, and (d) corresponds to an estimate of the case expected for a soft actuator, based on a low-order dynamic fit to the transmission characteristics shown in Fig. 1. In the latter case, the residual vibration is negligible, as the transmission from the mirror cell through to the segment is less than one.

\section{RESPONSE SUMMARY}

The information above has been used in TMT's actuator downselect process. Various different actuator technologies have been considered, prototyped, and characterized. ${ }^{9}$ The analysis below summarizes this process for a soft (voice-coil) actuator with a collocated high-bandwidth servo loop, and for a hard actuator with parameters representative of one of the options considered. A hard actuator is not viable without some added damping, due to the resulting vibration response, however, a collocated force-feedback loop can be used to add damping to a high-impedance position actuator, and this is assumed here.

The assumptions on wind and vibration parameters, and on the actuator options are summarized in Table 1, and the corresponding response given in Table 2. The performance evaluation for each actuator weighted both the nominal performance and the performance robustness to the following worst-case scenarios:

1. Worst-case wind model (including additional small-scale turbulence to account for possible unmodeled effects in the CFD),

2. Worst-case vibration amplitudes (doubling the assumed disturbance level, i.e. to a disturbance that yields the same M1 amplitude measured at Keck when Keck segment parameters are assumed in the TMT model),

3. Worst-case assumptions on actuators; for the soft actuator this means lower servo integral gain, for the hard actuator this means lower added damping,

4. Worst-case assumptions on the telescope structure, including lower damping ratio and $5 \%$ less SSA stiffness and

5. A "cross-term" where it was assumed that two assumptions were simultaneously changed to their worstcase values in order to account for the weakest performance category for each actuator; for the soft actuator this means worst wind assumptions and a low servo gain, while for the hard actuator this means worst vibration assumptions and lower added damping. 


\begin{tabular}{|c|c|c|c|}
\hline \multicolumn{2}{|c|}{ Parameter } & Nominal & Worst-case \\
\hline \multirow{3}{*}{ Wind } & mean wind speed & $1 \mathrm{~m} / \mathrm{s}$ & \\
& rms pressure & $0.21 \mathrm{~Pa}$ & $0.25 \mathrm{~Pa}$ \\
& $\beta$ & 0 & 0.2 \\
\hline \multirow{2}{*}{ Vibration } & frequency & $29.6 \mathrm{~Hz}$ & \\
& Amplitude & $13 \mathrm{~nm}$ & $26 \mathrm{~nm}$ \\
\hline \multirow{2}{*}{ Structure } & SSA stiffness scaling & 1.0 & 0.95 \\
& Structure damping & $0.5 \%$ & $0.25 \%$ \\
\hline \multirow{2}{*}{ Soft actuator } & integral gain & $2.2 \times 10^{7} \mathrm{~N} /(\mathrm{ms})$ & $1.1 \times 10^{7} \mathrm{~N} /(\mathrm{ms})$ \\
& static stiffness & $11.4 \mathrm{~N} / \mu \mathrm{m}$ & \\
\hline \multirow{2}{*}{ Hard actuator } & static stiffness & $60 \mathrm{~N} / \mu \mathrm{m}$ & \\
& high freq. stiffness & $8.5 \mathrm{~N} / \mu \mathrm{m}$ & \\
& damping & $10 \%$ & $6 \%$ \\
\hline
\end{tabular}

Table 1. Key parameter assumptions that influence performance calculations; worst-case assumptions are omitted for clarity if they are identical to the nominal values. For wind, $\beta$ is defined in eq. (1). The vibration amplitude specified is defined as the rms M1 surface vibration if segment and actuator parameters are the same as at Keck. Actuator static stiffness is the residual stiffness after an integral servo loop is closed, the high frequency stiffness of the hard actuator is the stiffness above the servo loop bandwidth.

\begin{tabular}{|c||c|c|c||c|c|c|}
\hline \multicolumn{1}{|c||}{} & \multicolumn{3}{c||}{ Soft actuator } & \multicolumn{3}{c|}{ Hard actuator } \\
& Wind & Vib & Combined & Wind & Vib & Combined \\
\hline Nominal & 0.9971 & 1.0000 & 0.9971 & 0.9984 & 0.9994 & 0.9978 \\
Worst wind & 0.9957 & & 0.9957 & 0.9977 & & 0.9971 \\
Worst vib & & 0.9998 & 0.9969 & & 0.9974 & 0.9958 \\
Worst actuator & 0.9949 & & 0.9949 & & 0.9988 & 0.9972 \\
Worst structure & 0.9969 & & 0.9969 & 0.9982 & 0.9985 & 0.9967 \\
"Cross-term" & 0.9921 & & 0.9921 & & 0.9953 & 0.9937 \\
\hline
\end{tabular}

Table 2. Performance (PSSN) corresponding to nominal and worst-case assumptions described in Table 1. Worst-case performance values are omitted for clarity if they are identical to the nominal values. The performance budget for the combination of these two sources is 0.9935 . The soft actuator provides better vibration rejection but lower wind rejection, but the difference in the combined performance is small compared to the uncertainties in the disturbance sources.

\section{CONCLUSIONS}

There are two main sources of dynamic disturbances on the telescope primary mirror; unsteady wind and narrowband equipment vibration. Understanding these sources is important for making design decisions. For example, the primary mirror actuator technology in particular influences the response, with soft actuators providing better attenuation to high frequency $(\sim 30 \mathrm{~Hz})$ equipment vibration, and hard actuators providing better stiffness for low frequency $(<1 \mathrm{~Hz})$ wind disturbances.

Models are presented for both of these sources. The wind response is a strong function of the assumed outer scale of turbulence over M1, and of the wind speed. Using potentially conservative assumptions on the outer scale, then with the $1 \mathrm{~Hz}$ bandwidth predicted to be achievable for the TMT primary mirror control system, the performance is acceptable even under worst-case assumptions on the wind or actuator parameters. Vibration estimates are anchored with data from the Keck Observatory, with the TMT vibration environment assumed to be a factor of two smaller. Acceptable performance is obtained with either a soft actuator, or a hard actuator with added damping.

The disturbance characterization and performance estimation supported TMT's actuator downselect process. This evaluated the response not only for nominal assumptions about the disturbance and actuator characteristics, but also for reasonable worst-case parameters in order to evaluate robustness to uncertainty. 


\section{Acknowledgements}

The finite element models used herein for the telescope structure and the primary segment assembly were developed by DSL and by HYTEC Inc., respectively. Actuator parameters used here are based on prototypes from Marjan Research and the Jet Propulsion Laboratory. The contributions of these organizations are gratefully acknowledged.

The TMT Project gratefully acknowledges the support of the TMT partner institutions. They are the Association of Canadian Universities for Research in Astronomy (ACURA), the California Institute of Technology and the University of California. This work was supported as well by the Gordon and Betty Moore Foundation, the Canada Foundation for Innovation, the Ontario Ministry of Research and Innovation, the National Research Council of Canada, the Natural Sciences and Engineering Research Council of Canada, the British Columbia Knowledge Development Fund, the Association of Universities for Research in Astronomy (AURA) and the U.S. National Science Foundation.

A portion of the research in this paper was carried out at the Jet Propulsion Laboratory, California Institute of Technology, under a contract with the National Aeronautics and Space Administration.

\section{REFERENCES}

1. MacMynowski, D. G., Vogiatzis, K., Angeli, G. Z., Fitzsimmons, J., and Nelson, J., "Wind Loads on GroundBased Telescopes," Applied Optics, Vol. 45, No. 30, pp. 7912-7923, 2006.

2. Cho, M. K., Stepp, L. M., Angeli, G. Z., and Smith, D. R., "Wind loading of large telescopes," Large Ground-Based Telescopes (Oschmann, and Stepp, eds.), 2002, pp. 352-367. Proc. SPIE 4837.

3. Angeli, G. Z., Cho, M. K., Sheehan, M., and Stepp, L. M., "Characterization of Wind Loading of Telescopes," Proc. SPIE Vol. 4757; Workshop on Integrated Modeling of Telescopes (Andersen, T., ed.), 2002, pp. 72-83.

4. MacMynowski, D. G., Blaurock, C., and Angeli, G. Z., "Dynamic Analysis of TMT," Proc. SPIE, 2008. SPIE 7017-31.

5. Seo, B.-Y., Nissly, C. R., Angeli, G. Z., Ellerbroek, B. L., Nelson, J. E., Sigrist, N., and Troy, M., "Analysis of Point Source Sensitivity as a performance metric for the Thirty Meter Telescope," Proc. SPIE, 2008. SPIE 7017-28.

6. Angeli, G. Z., Roberts, S., and Vogiatzis, K., "Systems engineering for the Thirty Meter Telescope," SPIE $7738-45,2010$.

7. MacMynowski, D. G. and Andersen, T., "Wind buffeting of large telescopes," Applied Optics, Vol. 49, No. 4, pp. $625-636,2010$.

8. MacMynowski, D. G., Thompson, P., Shelton, C., and Roberts, Jr., L. C., "Robustness of Thirty Meter Telescope primary mirror control," Proc. SPIE 7733, 2010.

9. Thompson, P. M., MacMynowski, D. G., Colavita, M. M., Regehr, M. W., and Sirota, M. J., "Servo design and analysis for the Thirty Meter Telescope primary mirror actuators," SPIE Vol. 7733, 2010. 